\title{
On multiple zeros of Bernoulli polynomials
}

by

\author{
KARL Dilcher (Halifax)
}

1. Introduction. The Bernoulli polynomials $B_{n}(x)$ can be defined by the generating function

$$
\frac{t e^{x t}}{e^{t}-1}=\sum_{n=0}^{\infty} B_{n}(x) \frac{t^{n}}{n !}, \quad|t|<2 \pi,
$$

or equivalently by way of the Bernoulli numbers defined by

$$
\frac{t}{e^{t}-1}=\sum_{n=0}^{\infty} B_{n} \frac{t^{n}}{n !}, \quad|t|<2 \pi,
$$

and the relation

$$
B_{n}(x)=\sum_{j=0}^{n}\left(\begin{array}{l}
n \\
j
\end{array}\right) B_{j} x^{n-j}
$$

for all integers $n \geq 0$. Since (1.2) shows that $B_{0}=1$, and an easy recurrence relation shows that all $B_{n}$ are rational numbers, the identity (1.3) tells us that the $n$th Bernoulli polynomial $B_{n}(x)$ is monic of degree $n$ and has rational coefficients.

Bernoulli polynomials have important applications in different areas of mathematics, most notably in number theory, asymptotic analysis, and the theory of finite differences. Many of these applications have their origins in the fundamental difference equation

$$
B_{n}(x+1)-B_{n}(x)=n x^{n-1}, \quad n \geq 1,
$$

which is easy to obtain by manipulating the generating function (1.1). Numerous other properties can be found, e.g., in [1, Ch. 23], [9], or [14]; for a comprehensive bibliography, see [8].

2000 Mathematics Subject Classification: Primary 11B68; Secondary 12D10.

Key words and phrases: Bernoulli polynomials, zeros, Bernoulli numbers, combinatorial sums.

Research supported in part by the Natural Sciences and Engineering Research Council of Canada. 
Among the different aspects of Bernoulli polynomials, their zeros have also been studied quite extensively, both from an analytic and an algebraic point of view. For a brief summary of results, see [6]. One question that has been asked is that of the possibility of multiple zeros. While this is interesting in its own right, zero multiplicity properties of Bernoulli and related polynomials have been used in the study of certain diophantine equations involving sums of powers; see, e.g., [10], [15], or [4].

Along with a detailed study of various algebraic properties of Bernoulli polynomials, Brillhart [3] established the following result on multiple zeros of Bernoulli polynomials.

Theorem 1 (Brillhart, 1969).

(a) $B_{2 m+1}(x)$ has no multiple zeros for any $m \geq 0$.

(b) Any multiple zero of $B_{2 m}(x)$ must be a zero of $x^{2}-x-b$, with $b$ a positive odd integer.

It appears that no further work on this problem has been published. It is the purpose of this paper to fill the gap left by Brillhart's result and to use the strong condition given in part (b) of his theorem, to prove

TheOrem 2. Bernoulli polynomials have no multiple zeros.

In Section 2 the reader will be reminded of some important properties of Bernoulli numbers and polynomials that are necessary for the proof, and some auxiliary results on combinatorial sums will be quoted or derived. Section 3 contains the proof of Theorem 2, and we conclude the paper with some additional remarks in Section 4.

2. Auxiliary results. We begin by quoting some basic properties of Bernoulli polynomials, for easy reference. First, it is obvious from (1.3) or (1.1) that $B_{n}(0)=B_{n}$, and by (1.4) we also have $B_{n}(1)=B_{n}$ for $n \geq 2$. By manipulating the generating functions (1.1) and (1.3), we get

$$
B_{n}(1 / 2)=\left(2^{1-n}-1\right) B_{n},
$$

and together with the fact that $B_{2 n+1}=0$ for all $n \geq 1$, we immediately see that $0,1 / 2$, and 1 are zeros of $B_{2 n+1}(x)$. In fact, it was shown by Inkeri [13] that these are the only rational zeros of any Bernoulli polynomial; see also $[3$, p. 45, 59]. Another important property is that the derivative of the $n$th Bernoulli polynomial is, essentially, the $(n-1)$ st, namely

$$
B_{n}^{\prime}(x)=n B_{n-1}(x), \quad n \geq 1 .
$$

In other words, the Bernoulli polynomials form an Appell sequence. This identity follows easily from (1.1), by taking the derivative with respect to $x$ on both sides of the identity. Following Brillhart [3], we use (2.1) and (2.2) 
to expand $B_{n}(x)$ about $x=1 / 2$, and obtain

$$
B_{n}(x)=\sum_{j=0}^{n}\left(\begin{array}{l}
n \\
j
\end{array}\right)\left(2^{1-j}-1\right)(x-1 / 2)^{n-j} B_{j},
$$

and since the only nonzero odd-index Bernoulli number is $B_{1}=-1 / 2$, we have

$$
B_{n}(x)=\sum_{j=0}^{\lfloor n / 2\rfloor}\left(\begin{array}{c}
n \\
2 j
\end{array}\right)\left(2^{1-2 j}-1\right)(x-1 / 2)^{n-2 j} B_{2 j},
$$

and this shows that $B_{n}(x+1 / 2)$ is an even or an odd function, depending on the parity of $n$.

The proof of Theorem 2 also requires one of the most important properties of the Bernoulli numbers, namely the theorem of von Staudt and Clausen (see, e.g., [11, p. 91]), here given in two of a variety of possible formulations.

Theorem 3 (von Staudt and Clausen).

(a) A prime $p$ divides the denominator of $B_{2 n}$ if and only if $p-1 \mid 2 n$.

(b) If $p-1 \mid 2 n$, then $p B_{2 n} \equiv-1(\bmod p)$.

It will become clear in the proof of Theorem 2 that the nonvanishing modulo $p$ of certain lacunary sums and alternating sums of binomial coefficients is required. For this purpose we need the following results.

LEMma 1 (Hermite and Bachmann). Let $p$ be a prime and $k$ a positive integer. Then

$$
\sum_{0<j(p-1)<k}\left(\begin{array}{c}
k \\
j(p-1)
\end{array}\right) \equiv 0(\bmod p) .
$$

In particular, for a positive integer $q$,

$$
\sum_{j=1}^{q}\left(\begin{array}{c}
q(p-1) \\
j(p-1)
\end{array}\right) \equiv 1(\bmod p) .
$$

The congruence (2.4) was first proved by Hermite [12] for odd integers $k$, and then extended by Bachmann $[2$, p. 46] to the general case. The alternating analogue to $(2.5)$ is

Lemma 2. Let $p$ be a prime and $q$ a positive integer. Then

$$
\sum_{j=1}^{q}(-1)^{j}\left(\begin{array}{cl}
q(p-1) \\
j(p-1)
\end{array}\right) \equiv \begin{cases}-1(\bmod p) & \text { for } q \text { odd }, \\
1(\bmod p) & \text { for } q \text { even }, p+1 \nmid q, \\
0(\bmod p) & \text { for } p+1 \mid q .\end{cases}
$$

This is the main result in [7]. Finally, we require a variant of the congruence (2.6). 
Lemma 3. Let $p \geq 3$ be a prime and $q \geq 2$ an even integer. Then

$$
\sum_{j=1}^{q-1}(-1)^{j}\left(\begin{array}{cl}
q(p-1)-1 \\
j(p-1)
\end{array}\right) \equiv \begin{cases}(p-1) / 2(\bmod p) & \text { when } p+1 \mid q, \\
0(\bmod p) & \text { otherwise. }\end{cases}
$$

Proof. Using standard properties of binomial coefficients, we have

$$
\begin{aligned}
\left(\begin{array}{c}
q(p-1) \\
j(p-1)
\end{array}\right) & =\left(\begin{array}{l}
q(p-1)-1 \\
j(p-1)-1
\end{array}\right)+\left(\begin{array}{c}
q(p-1)-1 \\
j(p-1)
\end{array}\right) \\
& =\frac{j}{q}\left(\begin{array}{c}
q(p-1) \\
j(p-1)
\end{array}\right)+\left(\begin{array}{c}
q(p-1)-1 \\
j(p-1)
\end{array}\right),
\end{aligned}
$$

and thus

$$
\left(\begin{array}{c}
q(p-1)-1 \\
j(p-1)
\end{array}\right)=\frac{q-j}{q}\left(\begin{array}{c}
q(p-1) \\
j(p-1)
\end{array}\right)
$$

With this identity we get

$$
\begin{aligned}
\sum_{j=1}^{q-1}(-1)^{j}\left(\begin{array}{c}
q(p-1)-1 \\
j(p-1)
\end{array}\right) & =\frac{1}{q} \sum_{j=1}^{q-1}(-1)^{j}(q-j)\left(\begin{array}{c}
q(p-1) \\
j(p-1)
\end{array}\right) \\
& =\frac{1}{q} \sum_{j=1}^{q-1}(-1)^{q-j} j\left(\begin{array}{c}
q(p-1) \\
j(p-1)
\end{array}\right)
\end{aligned}
$$

where we have switched the order of summation and used the fact that $\left(\begin{array}{c}n \\ n-k\end{array}\right)=\left(\begin{array}{l}n \\ k\end{array}\right)$. Now, if $q$ is even, then by adding the two expressions on the right of (2.8) we obtain

$$
2 \sum_{j=1}^{q-1}(-1)^{j}\left(\begin{array}{c}
q(p-1)-1 \\
j(p-1)
\end{array}\right)=\sum_{j=1}^{q}(-1)^{j}\left(\begin{array}{c}
q(p-1) \\
j(p-1)
\end{array}\right)-1,
$$

and the congruence (2.7) follows immediately from the second and third parts of (2.6).

3. Proof of Theorem 2. By Brillhart's result (Theorem 1) we may restrict our attention to even-index Bernoulli polynomials, and we know that any multiple zero of $B_{2 m}(x)$ must be a zero of $x^{2}-x-b$, with $b$ a positive integer. Let $x_{b}$ be such a zero, and assume it is a multiple zero of $B_{2 m}(x)$ for a fixed $m$. Then it is also a zero of $B_{2 m-1}(x)$. Our goal is to lead this to a contradiction.

We begin by noting that

$$
4\left(x_{b}-1 / 2\right)^{2}=4 x_{b}^{2}-4 x_{b}+1=4 b+1 .
$$


Then by (2.3) we have

$$
\begin{aligned}
2^{2 m} B_{2 m}\left(x_{b}\right) & =\sum_{j=0}^{m}\left(\begin{array}{c}
2 m \\
2 j
\end{array}\right)(4 b+1)^{m-j}\left(2-2^{2 j}\right) B_{2 j}, \\
2^{2 m-1} \sqrt{4 b+1} B_{2 m-1}\left(x_{b}\right) & =\sum_{j=0}^{m-1}\left(\begin{array}{c}
2 m-1 \\
2 j
\end{array}\right)(4 b+1)^{m-j}\left(2-2^{2 j}\right) B_{2 j} .
\end{aligned}
$$

We first consider the right-hand side of (3.1). Let $p$ be an odd prime such that $p-1 \mid 2 m$. We distinguish between three cases.

(i) Suppose that we can find such a prime $p$ with the additional property that $p \mid 4 b+1$. Then all the terms on the right of (3.1), with the exception of the last one (for $j=m$ ), are divisible by $p$ or, when $p=3$ and $j=m-1$, are $p$-integral. Hence we get from (3.1),

$$
p 2^{2 m} B_{2 m}\left(x_{b}\right) \equiv\left(2-2^{2 m}\right) p B_{2 m}(\bmod p) .
$$

Now, by Fermat's little theorem we have

$$
2^{2 m} \equiv 1(\bmod p) \quad \text { and } \quad 2-2^{2 m} \equiv 1(\bmod p) .
$$

With the theorem of von Staudt and Clausen (Theorem 3(b)) we then get

$$
p B_{2 m}\left(x_{b}\right) \equiv-1(\bmod p),
$$

which means that $B_{2 m}\left(x_{b}\right)$ cannot vanish.

(ii) Suppose that we have in fact $p-1=2 m$. Then by the von StaudtClausen theorem all terms on the right-hand side of (3.1), for $0 \leq j<m$, are $p$-integral, while the final term $\left(2-2^{p-1}\right) p B_{p-1}$ has $p$ in the denominator, which is not cancelled since $2^{p-1} \equiv 1(\bmod p)$. This means that $B_{2 m}\left(x_{b}\right)$ cannot vanish for any $b$.

(iii) Suppose there is no odd prime satisfying cases (i) or (ii); so let $p$ be an odd prime with $p-1 \mid 2 m$ but $p-1<2 m$ and $p \nmid 4 b+1$. Now set

$$
q=\frac{2 m}{p-1} ; \quad \text { then } \quad q \in \mathbb{Z}, 2 \leq q \leq m .
$$

Multiply both sides of (3.1) by $p$, and note that by the von Staudt-Clausen theorem we have

$$
p B_{2 j} \equiv \begin{cases}-1(\bmod p) & \text { when } 2 j=r(p-1), r=1,2, \ldots, q, \\ 0(\bmod p) & \text { for all other } j .\end{cases}
$$

Furthermore, for $2 j=r(p-1)$ we have by Fermat's little theorem,

$$
2-2^{2 j} \equiv 1(\bmod p),
$$


and since $p \nmid 4 b+1$,

$$
(4 b+1)^{j}=\left((4 b+1)^{(p-1) / 2}\right)^{r} \equiv \varepsilon_{b}^{r}(\bmod p),
$$

where by Euler's criterion we have

$$
\varepsilon_{b}= \begin{cases}1 & \text { if } 4 b+1 \text { is a quadratic residue }(\bmod p), \\ -1 & \text { otherwise }\end{cases}
$$

With (3.3), (3.4), and (3.5), the identity (3.1) gives us the congruence

$$
p B_{2 m}\left(x_{b}\right) \equiv-\varepsilon_{b}^{q} \sum_{r=1}^{q}\left(\begin{array}{c}
q(p-1) \\
r(p-1)
\end{array}\right) \varepsilon_{b}^{r}(\bmod p) .
$$

When $\varepsilon_{b}=1$, then by the congruence (2.5) we have

$$
p B_{2 m}\left(x_{b}\right) \equiv-1(\bmod p),
$$

and so $x_{b}$ cannot be a zero. When $\varepsilon_{b}=-1$, then Lemma 2 shows that

$$
p B_{2 m}\left(x_{b}\right) \equiv \begin{cases}0(\bmod p) & \text { when } p+1 \mid q, \\ \pm 1(\bmod p) & \text { otherwise. }\end{cases}
$$

Thus the only case that remains open is the case $p+1 \mid q$ and $\varepsilon_{b}=-1$, with the other conditions under (iii) above.

To deal with this case, we use the fact that if $x_{b}$ is a multiple zero of $B_{2 m}(x)$, it must be a zero of $B_{2 m-1}(x)$. Let $\widetilde{B}_{2 m-1}(x)$ be the right-hand side of (3.2). Then with the congruences (3.3), (3.4), and (3.5), and with $\varepsilon_{b}=-1$ we get, as above,

$$
\begin{aligned}
p \widetilde{B}_{2 m-1}\left(x_{b}\right) & \equiv-(-1)^{q} \sum_{r=1}^{q-1}(-1)^{r}\left(\begin{array}{c}
q(p-1)-1 \\
r(p-1)
\end{array}\right)(\bmod p) \\
& \equiv(-1)^{q-1} \frac{p-1}{2}(\bmod p)
\end{aligned}
$$

when $p+1 \mid q$, where the last congruence follows from Lemma 3 . This means that $B_{2 m-1}\left(x_{b}\right)$ cannot be zero, which completes the proof of Theorem 2 .

4. Additional remarks. Closely related to the Bernoulli polynomials are the Euler polynomials $E_{n}(x)$ which can be defined by the generating function

$$
\frac{2 e^{x t}}{e^{t}+1}=\sum_{n=0}^{\infty} E_{n}(x) \frac{t^{n}}{n !}, \quad|t|<\pi ;
$$

see, e.g., [1, Ch. 23]. It was observed by Carlitz [5] that

$$
E_{5}(x)=(x-1 / 2)\left(x^{2}-x-1\right)^{2},
$$

so that there are indeed multiple zeros. However, Brillhart [3] showed that no other Euler polynomial has multiple zeros. 


\section{References}

[1] M. Abramowitz and I. A. Stegun, Handbook of Mathematical Functions, National Bureau of Standards, 1964.

[2] P. Bachmann, Niedere Zahlentheorie. Zweiter Teil, B. G. Teubner, Leipzig, 1910. Reprinted as two volumes in one, Chelsea, New York, 1968.

[3] J. Brillhart, On the Euler and Bernoulli polynomials, J. Reine Angew. Math. 234 (1969), 45-64.

[4] B. Brindza, On some generalizations of the diophantine equation $1^{k}+2^{k}+\cdots+x^{k}$ $=y^{z}$, Acta Arith. 44 (1984), 99-107.

[5] L. Carlitz, Note on irreducibility of the Bernoulli and Euler polynomials, Duke Math. J. 19 (1952), 475-481.

[6] K. Dilcher, Zeros of Bernoulli, generalized Bernoulli and Euler polynomials, Mem. Amer. Math. Soc. 73 (1988), no. 386.

[7] —, Congruences for a class of alternating sums of binomial coefficients, J. Integer Seq. 10 (2007), Art. 07-10.1, 10 pp.

[8] K. Dilcher, L. Skula, and I. Sh. Slavutskii, Bernoulli Numbers. Bibliography (17131990), Queen's Papers Pure Appl. Math. 87, Queen's Univ., Kingston, Ont., 1991. Updated on-line version: http://www.mathstat.dal.ca/ dilcher/bernoulli.html.

[9] R. L. Graham, D. E. Knuth, and O. Patashnik, Concrete Mathematics, 2nd ed., Addison-Wesley, Reading, MA, 1994.

[10] K. Győry, R. Tijdeman, and M. Voorhoeve, On the equation $1^{k}+2^{k}+\cdots+x^{k}=y^{z}$, Acta Arith. 37 (1980), 233-240.

[11] G. H. Hardy and E. M. Wright, An Introduction to the Theory of Numbers, 5th ed., Oxford Univ. Press, 1979.

[12] Ch. Hermite, Extrait d'une lettre à M. Borchardt (sur les nombres de Bernoulli), J. Reine Angew. Math. 81 (1876), 93-95.

[13] K. Inkeri, The real roots of Bernoulli polynomials, Ann. Univ. Turku. Ser. AI 37 (1959), $20 \mathrm{pp}$.

[14] N. E. Nörlund, Vorlesungen über Differenzenrechnung, Springer, Berlin, 1924.

[15] M. Voorhoeve, K. Györy, and R. Tijdeman, On the Diophantine equation $1^{k}+\cdots+$ $x^{k}+R(x)=y^{z}$, Acta Math. 143 (1979), 1-8.

Department of Mathematics and Statistics

Dalhousie University

Halifax, Nova Scotia, B3H 3J5, Canada

E-mail: dilcher@mathstat.dal.ca

Received on 6.12.2007

and in revised form on 17.1.2008 